\title{
Visualization of individual carbon nanotubes with fluorescence microscopy using conventional fluorophores
}

\author{
Rohit Prakash \\ Department of Physics and Astronomy, University of North Carolina, Chapel Hill, North Carolina 27599 \\ S. Washburn and Richard Superfine \\ Department of Physics and Astronomy, Curriculum for Applied and Materials Science, \\ Department of Computer Science, University of North Carolina, Chapel Hill, North Carolina 27599 \\ Richard E. Cheney \\ Department of Cell and Molecular Physiology, University of North Carolina, \\ Chapel Hill, North Carolina 27599 \\ Michael R. Falvo a) \\ Curriculum for Applied and Materials Science, University of North Carolina, \\ Chapel Hill, North Carolina 27599
}

(Received 25 April 2003; accepted 9 June 2003)

\begin{abstract}
We demonstrate that individual carbon nanotubes (CNTs) can be visualized with fluorescence microscopy through noncovalent labeling with conventional fluorophores. Reversal of contrast in fluorescence imaging of the CNTs was observed when performing labeling procedure in a nonpolar solvent. Our results are consistent with a CNT-fluorophore affinity mediated by hydrophobic interaction. The reverse-contrast images also provide clear indication of nanotube location. (C) 2003 American Institute of Physics. [DOI: 10.1063/1.1599042]
\end{abstract}

There have been several reports on efforts to attach molecules and proteins to carbon nanotubes (CNTs) through covalent $^{1-4}$ as well as noncovalent interactions. ${ }^{5-8}$ In some of these, the goal of specific immobilization of biological molecules on CNTs for biological and chemical sensing applications $^{7,9}$ was explored. Other work has focused on optical properties of molecules adsorbed onto CNTs. In these studies, optical behavior of adsorbed molecules was used both as a mode of probing molecule/CNT interactions ${ }^{2}$ as well as a method of labeling the CNTs for fluorescence microscopy. ${ }^{5}$ Unlike covalent functionalization, which requires damage to the atomic lattice and the electronic properties of the CNT, labeling through noncovalent attachments allows for the entire length of the CNTs to be visualized without perturbing the $s p^{2}$ bonded graphene sidewall. Like many biological structures studied with optical microscopy, CNTs are too small to observe consistently without the aid of fluorescent tags. Larger CNTs can be imaged with phase or differential interference contrast imaging with the help of video enhancement, but reliable fluorescence labeling allows for much smaller CNTs to be visualized with optical microscopy. We present here a preliminary study of the noncovalent binding of several common fluorescent dyes to multiwall CNTs in both polar and nonpolar solutions. These results complement recent reports of attachment of a fluorescent polymer to single-wall CNTs and subsequent imaging of single-wall CNT bundles in a polar solvent $(1,2$ dicholorethane). ${ }^{5}$ This is a report of fluorescence imaging of individual CNTs in biologically relevant environments.

We labeled the CNTs using two procedures. In method 1, CNTs were deposited from suspension onto glass slides and

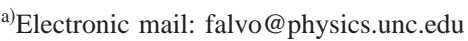

the solvent removed by evaporation upon spin coating. The flourophore solution was prepared by adding $1.5 \mu \mathrm{l}$ of the fluorophore solution $(5 \mathrm{mg} / \mathrm{ml}$ in $100 \%$ methanol) to $99 \mu \mathrm{l}$ of diH20. This solution was then passed over the adsorbed CNT in a simple flow cell mounted in an optical microscope. In method 2, $250 \mu$ of the CNT suspension was combined with the fluorophore/water solution in a test tube and mixed for 5 $\mathrm{s}$ prior to spin coating onto a glass slide for imaging (spun $350 \mu \mathrm{l}$ in seven $50 \mu \mathrm{l}$ aliquots onto glass slide). For both methods, the CNT suspensions were prepared by sonicating $\sim 1 \mathrm{mg}$ of arc prepared CNT soot in $20 \mathrm{ml}$ of methanol.

Two optical microscopes were used for this work: a Zeiss Axiovert 100TV with Hamamatsu Orca-II cooled charge coupled device (CCD) camera (Figs. 2 and 3), and a Nikon inverted Diaphot 200 with Spot 2 cooled-CCD camera (Fig. 4). Samples were imaged using phase contrast, differential interference contrast (DIC) and fluorescence imaging. A tetramethyl rhodamine isothiocyanate filter cube was used for all fluorescence imaging, (excitation filter 530-560 nm, dichroic mirror wavelength $570 \mathrm{~nm}$, barrier filter 590-650 $\mathrm{nm})$. The following dyes were used in this study:

(1) $\operatorname{DiIC}_{\mathbf{1 6}}(3) \quad 1,1^{\prime}$ - dihexadecyl-3,3,3',3' - tetramethylindocarbocyanine perchlorate (molecular probes D-384);

(2) $\mathbf{D i O C}_{6}(3)$ 3,3' -dihexyloxacarbocyanine iodide (molecular probes D-273);

(3) $\mathbf{D i O C}_{\mathbf{5}}(\mathbf{3})$ 3,3' -dipentyloxacarbocyanine iodide (molecular probes D-272);

(4) $\mathbf{D i O C}_{2}$ (3) 3,3' - diethyloxacarbocyanine iodide (molecular probes D-14730);

(5) BODIPY® FL $\mathbf{C}_{\mathbf{1 6}}$ 4,4-difluoro-5, 7-dimethyl-4-bora3a, 4a-diaza-s-indacene-3-hexadecanoic acid (molecular probes D-3821); and 
<smiles>CCN1C(=CC=CC2=[N+](CC)c3ccccc3C2(C)C)C(C)(C)c2ccccc2N1CC=CC=CC=CC=Cc1oc2ccccc2[n+]1C</smiles>

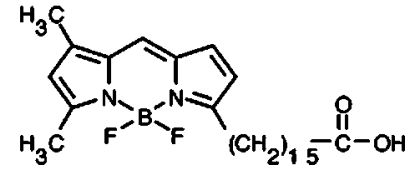

BODIPY

FIG. 1. Fluorophore structures.

(6) Alexa Fluor ${ }^{\circledR} \mathbf{4 8 8}$ hydrazide, sodium salt (molecular probes A-10436).

Of the six fluorophores tested in this study we saw successful labeling of CNTs with only the $\mathrm{DiIC}_{16}$ and the $\mathrm{DiOC}_{6}$. We saw very limited success with $\mathrm{DiOC}_{5}$ and no labeling at all with $\mathrm{DiOC}_{2}$, BODIPY or hydrazide. Here we define successful labeling as that which produced sufficient imaging contrast relative to background to locate CNTs in fluorescence images (where the CNT is bright relative to the background). The molecular structure of the two successful and one of the unsuccessful fluorophores are shown in Fig. 1. $\mathrm{DiOC}_{5}$ and $\mathrm{DiOC}_{2}$ have identical structure to $\mathrm{DiOC}_{6}$ aside from the number of methylene groups in the sidechains. The hyrodzide fluorophore is similar to BODIPY in that it has mostly polar moieties extending from the heterocyclic portion of the molecule.

In Fig. 2, CNTs are clearly visible in both the phase contrast image (top) and labeled with $\mathrm{DiOC}_{6}$ in the fluorescence image (bottom). The dye labeling was performed using method 1 as described earlier. In some trials, atomic force microscopy (AFM) was performed on samples to verify that CNTs observed in the optical images were individual CNT (Fig. 5). We found CNTs with diameters as small as $14 \mathrm{~nm}$ can be visualized with phase contrast and DIC imaging. Though we show no DIC images, we observed no systematic advantage of phase contrast versus DIC in imaging CNTs. In addition to the CNTs, carbonaceous debris was present, which is routinely seen accompanying CNT material. The dye readily labeled this material also, as is expected due to its graphitic composition. Figure 3 shows another case of CNTs labeled with dye (method 2). In this case the dye is DiIC $_{16}$. We note that since the CNTs are well below the diffraction limited resolution of the optical system, their apparent widths will be equal to the resolution limit in both the DIC and fluorescence images. ${ }^{10}$ In the case of our optical system, this limit will be roughly $300-600 \mathrm{~nm}$ depending on the numerical aperture values for the objective and condenser lenses.

The noncovalent attachment of the dye to the CNTs probably occurs through hydrophobic interaction. Graphene (the $s p^{2}$ bonded graphite plane) is hydrophobic. CNTs are essentially graphene sheets wrapped into seamless cylinders.

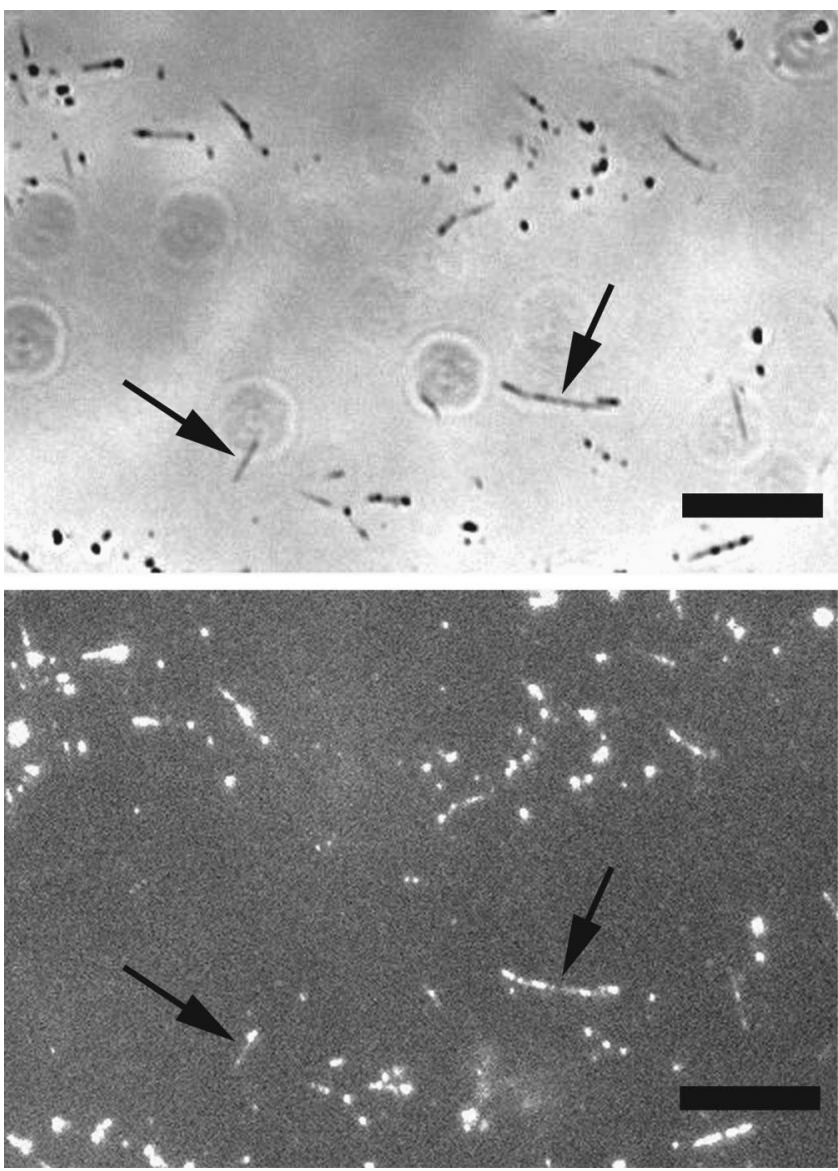

FIG. 2. Phase contrast image (top) and fluorescence image (bottom) of CNTs labeled with $\mathrm{DiOC}_{6}$. Scale bars are $10 \mu \mathrm{m}$ in length.

The dyes used in this study lack the polyaromatic structure that would best facilitate binding to the CNTs through $\pi$ stacking as has been suggested for molecules containing pyrene moieties. ${ }^{7}$ The success of the $\mathrm{DiIC}_{16}$ and the $\mathrm{DiOC}_{6}$ in contrast to the poor results of the $\mathrm{DiOC}_{5}$ and $\mathrm{DiOC}_{2}$ suggest that the binding was mediated through the hydrocarbon chains. The $\mathrm{DiIC}_{16}$ and $\mathrm{DiOC}_{6}$ having longer hydrophobic hydrocarbon chains, would bind more stably if the binding
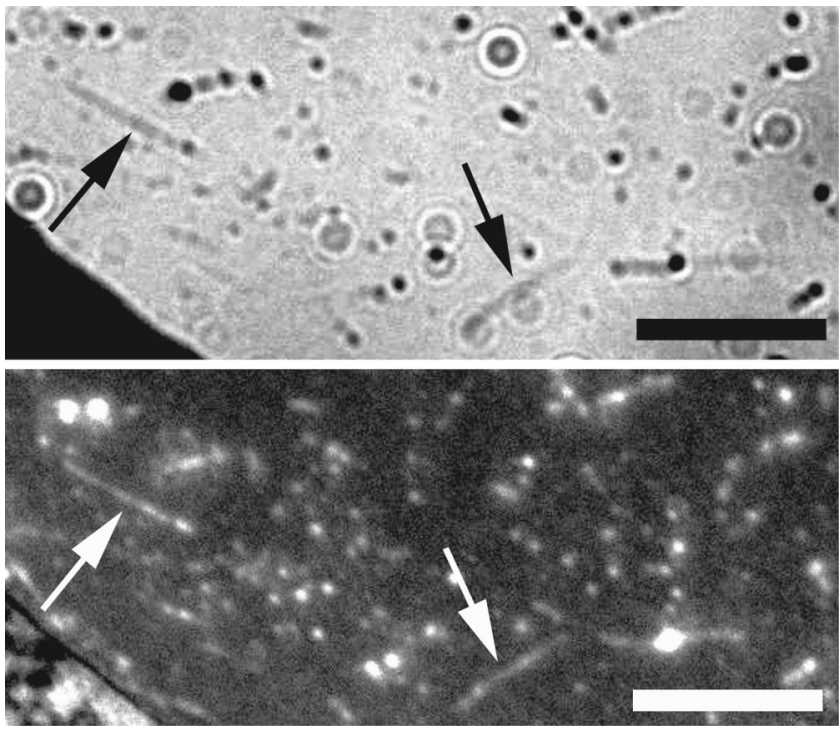

FIG. 3. Phase contrast (top) and fluorescence image (bottom) of CNTs labeled with $\mathrm{DiIC}_{16}$. Scale bars are $5 \mu \mathrm{m}$ in length. Downloaded 08 Aug 2003 to 152.2.131.62. Redistribution subject to AIP license or copyright, see http://ojps.aip.org/aplo/aplcr.jsp 


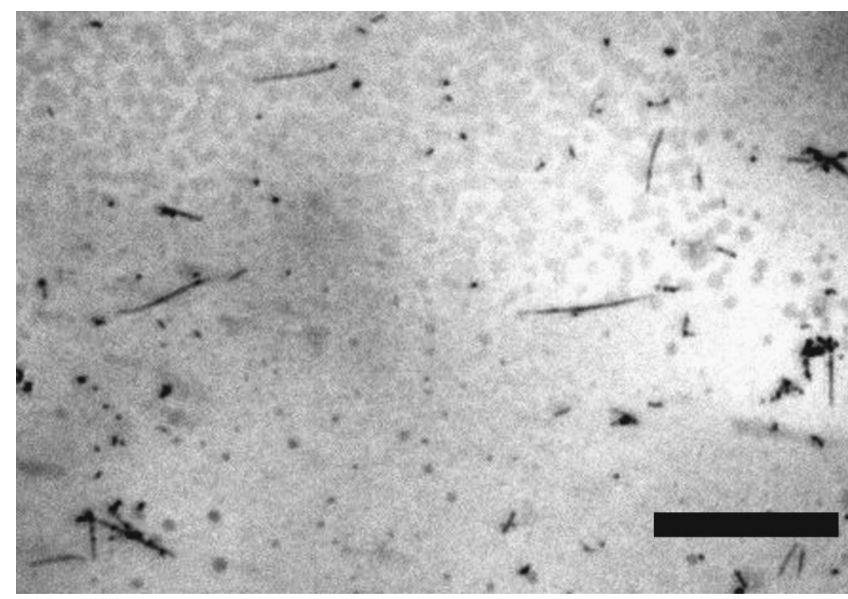

FIG. 4. Reversal of contrast in nonpolar solvent. Fluorescence image of CNTs labeled with DiIC $_{16}$. For this experiment, the CNTs and dye were mixed in a nonpolar solvent (DCE). Scale bar is $10 \mu \mathrm{m}$ in length.

happens through this part of the molecule (see Fig. 1).

To better elucidate the nature of the binding of the dye to the CNTs, we repeated the experiments using a nonpolar solvent (nonaqueous). The binding affinity of the dye to the CNTs in the nonpolar solvent should be significantly lower than in the aqueous case (assuming hydrophobic binding) because of the increased solubility of the dye in the solvent. Our results are consistent with this hypothesis. Figure 4 shows a fluorescence image of CNTs mixed with DiIC 16 (method 1) using 1,2-dichloroethane (DCE) in the prep instead of water. The fluorescence image shows a striking reversal of contrast when compared to the fluorescence images of Figs. 2 and 3. We interpret this image as indicating that the binding affinity of the fluorophore for the CNTs is lower than to the underlying glass substrate in the nonpolar solvent. We see a background of fluorescence from dye lying on the substrate and low fluorescence signal (implying less dye) coming from the CNTs.

Note that in these images, the intensities were adjusted for maximum contrast. The absolute fluorescence signal intensities indicate that the coverage of fluorophore on the underlying substrate was actually less for the DCE case than for the aqueous case, though the contrast and the brightness of the images imply otherwise. This suggests that the binding affinity of the fluorophore decreased for both the CNTs and the glass substrate when going from water to DCE. This decrease in affinity, however, was much more severe in the CNT case and we observed a reversal of contrast. Otobe et al. mention a similar solvent related contrast effect for single-walled CNT bundles in their report. ${ }^{5}$ As for the ab-

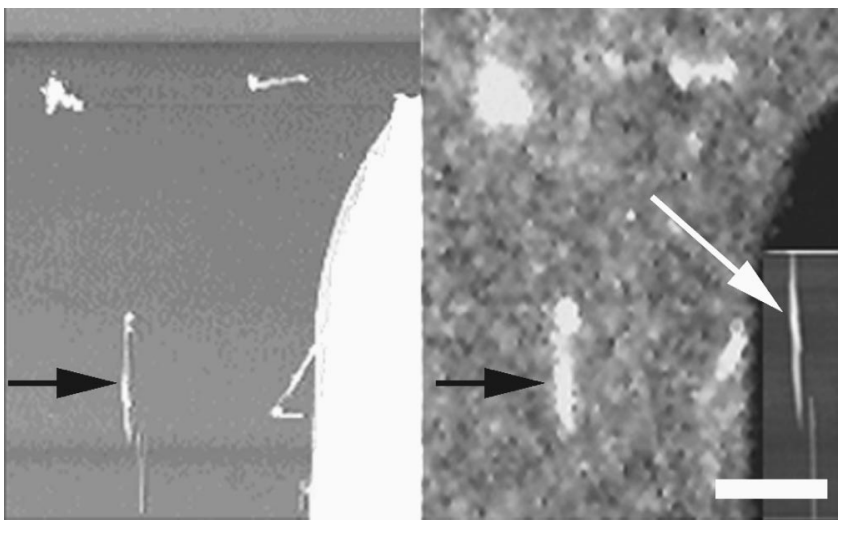

FIG. 5. AFM image of a CNT (left) before the addition of $\mathrm{DiOC}_{6}$. Fluorescence image (right) after the $\mathrm{DiOC}_{6}$ was added. Inset: AFM image of CNT after addition of $\mathrm{DiOC}_{6}$. The CNT indicated by the arrow was $18 \mathrm{~nm}$ in height before the addition of $\mathrm{DiOC}_{6}$, and $32 \mathrm{~nm}$ after. The scale bar is 5 $\mu \mathrm{m}$ in length. Note that the smaller CNT below the one indicated by the arrow is not labeled by the fluorophore. It is not clear to us why this is the case but we speculate it has to do with its small size. Further investigation will be needed to answer this question.

sence of quenching through energy transfer to the CNTs as has been reported, ${ }^{1-3}$ we briefly note that the AFM measurements were made of CNT before and after labeling for some trials to determine the thickness of the adsorbed dye (see Fig. 5). These data indicate a significant buildup of fluorophore $(>10 \mathrm{~nm})$ on the CNTs. The energy transfer was apparently sufficiently attenuated at these distances to allow for successful fluorescence imaging. ${ }^{5}$

The authors thank Tim O'Brien for helpful discussions on fluorescence microscopy and the National Science Foundation for supporting this work.

\footnotetext{
${ }^{1}$ V. Georgakilas, K. Kordatos, M. Prato, D. M. Guldi, M. Holzinger, and A. Hirsch, J. Am. Chem. Soc. 124, 760 (2002).

${ }^{2}$ L. W. Qu, R. B. Martin, W. J. Huang, K. F. Fu, D. Zweifel, Y. Lin, Y. P. Sun, C. E. Bunker, B. A. Harruff, J. R. Gord, and L. F. Allard, J. Chem. Phys. 117, 8089 (2002).

${ }^{3}$ J. E. Riggs, Z. X. Guo, D. L. Carroll, and Y. P. Sun, J. Am. Chem. Soc. 122, 5879 (2000).

${ }^{4}$ W. J. Huang, S. Taylor, K. F. Fu, Y. Lin, D. H. Zhang, T. W. Hanks, A. M. Rao, and Y. P. Sun, Nano Lett. 2, 311 (2002).

${ }^{5}$ K. Otobe, H. Nakao, H. Hayashi, F. Nihey, M. Yudasaka, and S. Iijima, Nano Lett. 2, 1157 (2002).

${ }^{6}$ M. Shim, N. W. S. Kam, R. J. Chen, Y. M. Li, and H. J. Dai, Nano Lett. 2, 285 (2002).

${ }^{7}$ R. J. Chen, Y. G. Zhan, D. W. Wang, and H. J. Dai, J. Am. Chem. Soc. 123, 3838 (2001).

${ }^{8}$ F. Balavoine, P. Schultz, C. Richard, V. Mallouh, T. W. Ebbesen, and C. Mioskowski, Angew. Chem., Int. Ed. 38, 1912 (1999).

${ }^{9}$ M. Shim, A. Javey, N. W. S. Kam, and H. J. Dai, J. Am. Chem. Soc. 123, 11512 (2001).

${ }^{10}$ D. B. Murphy, Fundamentals of Light Microscopy and Electronic Imaging
} (Wiley, New York, 2001). 